\title{
Schizophrenie
}

\section{Wider die Tabuisierung}

Frühe Diagnose und Therapie ermöglichen ein erfülltes und zufriedenes Leben.

\begin{abstract}
Wahnvorstellungen, ein beeinträchtigtes Gefühlsleben und Motivationsdefizite machen das Leben für Schizophreniepatienten und ihre Umwelt zu einer fast täglichen Herausforderung. Die große Unwissenheit über die Erkrankung macht die Situation zusätzlich kompliziert. Einer der schlimmsten Mythen, der sich um die Schizophrenieerkrankung rankt, ist die Assoziation, unheilbar - unbehandelbar". "Schizophrenie ist zwar nicht heilbar", so Univ.-Prof. Dr. Wolfgang Fleischhacker, Univ.-Klinik für Biologisch Psychiatrie, Innsbruck, „,sie ist aber gut behandelbar." Anlässlich des Internationalen Tages der seelischen Gesundheit

te sind nicht stabil, sie wechseln mit gesunden Phasen ab.

\section{Stigmatisierung und Ausgrenzung}

Schizophreniekranke leiden auch unter der gesellschaftlichen Reaktion auf ihre Erkrankung, erklärt Prim. Dr. Georg Psota, Präsident der Österreichischen Gesellschaft für Psychiatrie und Psychotherapie: Angst und soziale Ablehnung haben einer aktuellen Untersuchung zufolge in den vergangenen 20 Jahren sogar zugenommen. Stigmatisierung, soziale Ausgrenzung und Diskriminierung sind eine zusätzliche Belastung für die Betroffenen.
\end{abstract} stellte das Lundbeck Presseforum Psychiatrie Anfang Oktober "Leben mit Schizophrenie" zur Expertendiskussion. Die „gespaltene“ Persönlichkeit ist jedenfalls auch ein Mythos.

Die Symptome sind für den Betroffenen quälend: Die Vorstellung, das eigene Denken und Handeln werde von fremden Mächten beeinflusst, Halluzinationen vor allem im akustischen Bereich in Form von Stimmen, die Beschimpfungen von sich geben oder auch Befehle erteilen, Schwierigkeiten, Gefühle anderer Menschen wahrzunehmen oder zu verstehen, reduzierte Gedächtnisleistung und Aufmerksamkeit - die Realität wird verkannt, das eigene Leben gestaltet sich Aufgrund der Defizite häufig ziemlich desorganisiert und die Psychomotorik kann eingeschränkt sein. Die Vielfältigkeit der Ausprägung führt auch häufig zu Probleme in der Diagnose: Ist diese bei Auftreten von Wahnvorstellungen noch relativ einfach, so sind Symptome, die die emotionale und kognitive Ebene betreffen, leicht mit anderen psychischen Störungen wir Depressionen zu verwechseln. „Schizophrenie ist keine Persönlichkeitsstörung", stellt Fleischhacker fest, , aber die Persönlichkeit ist in vielerlei Weise gestört, beeinträchtigt und belastet." Doch die Defizi-

\section{Häufigkeit und Ursachen}

Schizophrenie ist eine Erkrankung, die in bei Männern und Frauen auftritt. Etwa ein Mensch unter 100 erkrankt irgendwann im Laufe seines Lebens an Schizophrenie, wobei der Beginn der Erkrankung meist im Adoleszenz-bzw. jungen Erwachsenenalter liegt. Dieser Zusammenfall mit einer ohnedies psychisch labilen Lebensphase führt auch dazu, dass die Diagnose oft verzögert gestellt wird. Gerade die frühe Diagnose und Therapie wären aber wichtig, um einen Krankheitsfortschritt mit immer wiederkehrenden Rückfällen und eine Chronifizierung zu vermeiden. Frühwarnzeichen, so Fleischhacker, „ist die Kontinuität der Symptome in der gesamten Fülle, die relativ rigide und unflexibel sind."

Die Ursachen für eine Schizophrenieerkrankung sind nicht vollständig aufgeklärt, zahlreiche Risikofaktoren, die einen Krankheitsausbruch begünstigen, sind jedoch bekannt. Dazu zählen biologische ebenso wie psychosoziale Aspekte. Eine genetische Belastung und eine Veränderung im Neurotransmitterhaushalt sind gut belegt. Aber auch die genetische Komponente ist von großer Vielfältigkeit geprägt: So sind mehr als 1000 Genmuallen Kulturkreisen und etwa gleich oft tationen bekannt, die das Risiko zu erkranken steigern. Schwere Erkrankungen der Mutter im zweiten Trimenon der Schwangerschaft oder perinatale Komplikationen führen zu einer erhöhten Verletzlichkeit, an einer Schizophrenie zu erkranken. Das Zusammenspiel von genetischer Belastung, vermehrter Dopaminempfindlichkeit und unterschiedlichsten Formen von chronischem Stress erhöht das Risiko. Da die letzte neuronale Reifung bis etwa zum 20. Lebensjahr erfolgt, kann das Auftreten von Stressoren hier als akuter Auslöser wirken.

\section{Medikamente und psychosoziale Maßnahmen}

Therapeutisch stehen eine Vielzahl an Antipsychotika, die regulierend in den Dopaminstoffwechsel eingreifen, und psychosoziale Therapiemaßnahmen zur Verfügung. Etwa $70 \%$ der früh behandelten Ersterkrankten erreichen nach drei bis sechs Monaten fast Symptomfreiheit, berichtet Fleischhacker: „Zur Aufrechterhaltung dieses Therapieerfolges ist zumeist eine langfristige prophylaktische antipsychotischer Therapie notwendig."

Eine gesellschaftliche Enttabuisierung soll auf beiden Seiten Angst und Unbehagen nehmen. Mit gezielter Öffentlichkeits- und Informationsarbeit wird versucht, der Öffentlichkeit Berührungsängste mit Betroffenen zu nehmen und eine möglichst frühe Therapie zu ermöglichen.,Durch frühe therapeutische Interventionen können heute viele Schizophreniekranke ein erfülltes, erfolgreiches und zufriedenes Leben führen", betonte Fleischhacker.

Dr. Verena Kienast

psychopraxis.neuropraxis 2015 · 18:64 DOI 10.1007/s00739-014-0220-3 Online publiziert: 27. November 2014 ๑) Springer-Verlag Wien 2014 